\title{
Chronic encapsulated intracerebral haematoma: pathogenetic and diagnostic considerations
}

\author{
ETTORE FIUMARA, MARCELLO GAMBACORTA, VINCENZO D'ANGELO, \\ MASSIMO FERRARA, CLAUDIO CORONA
}

From the Departments of Neurosurgery and Pathology, Niguarda Ca' Granda Hospital, Milan, Italy

SUMMARY Two cases of chronic encapsulated intracerebral haematoma are reported. The patients presented with progressive neurological deficits. Computed tomography scan showed a roundish, intracerebral lesion, that revealed ring blush after contrast infusion, with mass effect. At operation a thick, fibrous, brownish capsule, containing clots in different stages of formation, was removed. The hypothesis of capsule formation due to an exuberant proliferation of arachnoidal fibroblasts, is discussed. Differentiation between encapsulated intracerebral haematoma and other chronic intracerebral blood collections is considered mandatory for appropriate treatment.

Intracerebral haematoma (ICH) can usually be easily recognised from the clinical history, neurological examination and computed tomography (CT) findings. The natural evolution of the clots is by absorption, leaving a residual small scar or cyst.

A few patients in whom a chronic encapsulated $\mathrm{ICH}$ developed, simulating a tumour, have been reported. We present two further cases. We assess their pathogenesis, clinical, neuroradiological and surgical features. We also suggest a classification of chronic $\mathrm{ICH}$.

\section{Case reports}

Case 1

A 33 year old man presented in November 1983 with a four month history of repeated convulsions of the arm. In the two months before admission he suffered from numbness of his right side and progressive difficulty with speech. There was no history of head injury or arterial hypertension. Neurological examination revealed mild dysphasia, dyslexia, dysgraphia, and right sided hyperreflexia.

A CT scan showed a well demarcated, roundish, hyperdense lesion in the left parietal lobe with perifocal oedema and mass effect. The ring of the lesion slightly enhanced after contrast infusion (fig 1). Cerebral angiography revealed an avascular left parietal mass. At operation a well demarcated lesion with a thick, elastic, brownish capsule was found at a

Correspondence to: Ettore Fiumara, MD, via V. Foppa No 60, 20144 Milano, Italy.

Received 27 September 1988 and in revised form 28 January 1989. Accepted 18 March 1989 depth of about $2 \mathrm{~cm}$ from the cortex. A small amount of old, dark bloody fluid was aspirated by puncture and the capsule $\widetilde{\nabla}$ was totaliy removed. No pathological vessels were seen macroscopically. Postoperatively the patient experienced $\stackrel{\square}{\varrho}$ several episodes of convulsions of the right upper extremity and afterwards developed aphasia and right hemiplegia, which progressively and partially recovered. After three years follow up only a mild dysphasia was still present.

\section{Case 2}

A 64 year old man presented in January 1987 with a three month history of progressive confusion, disorientation and apathy. Seven months before admission he suddenly developed a visual defect of the right hemifield. A CT scan, performed one month after this episode, revealed only a

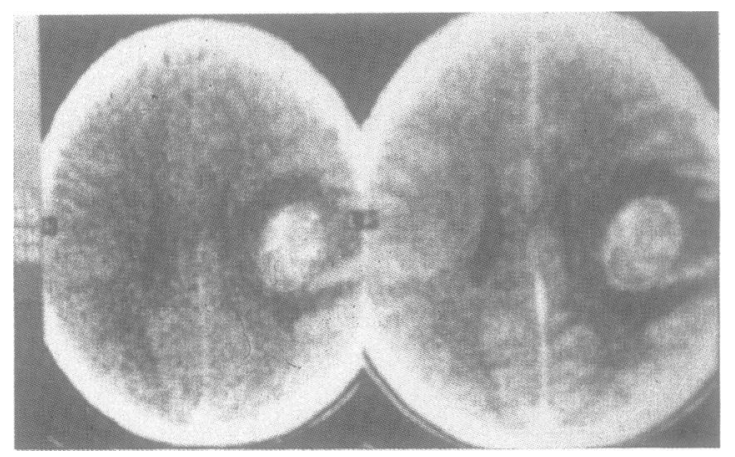

Fig 1 Plain CT scan showing a round hyperdense lesion in the left parietal lobe. Perifocal oedema and mass effect are present (left). The ring of the lesion enhances slightly with contrast infusion (right). 

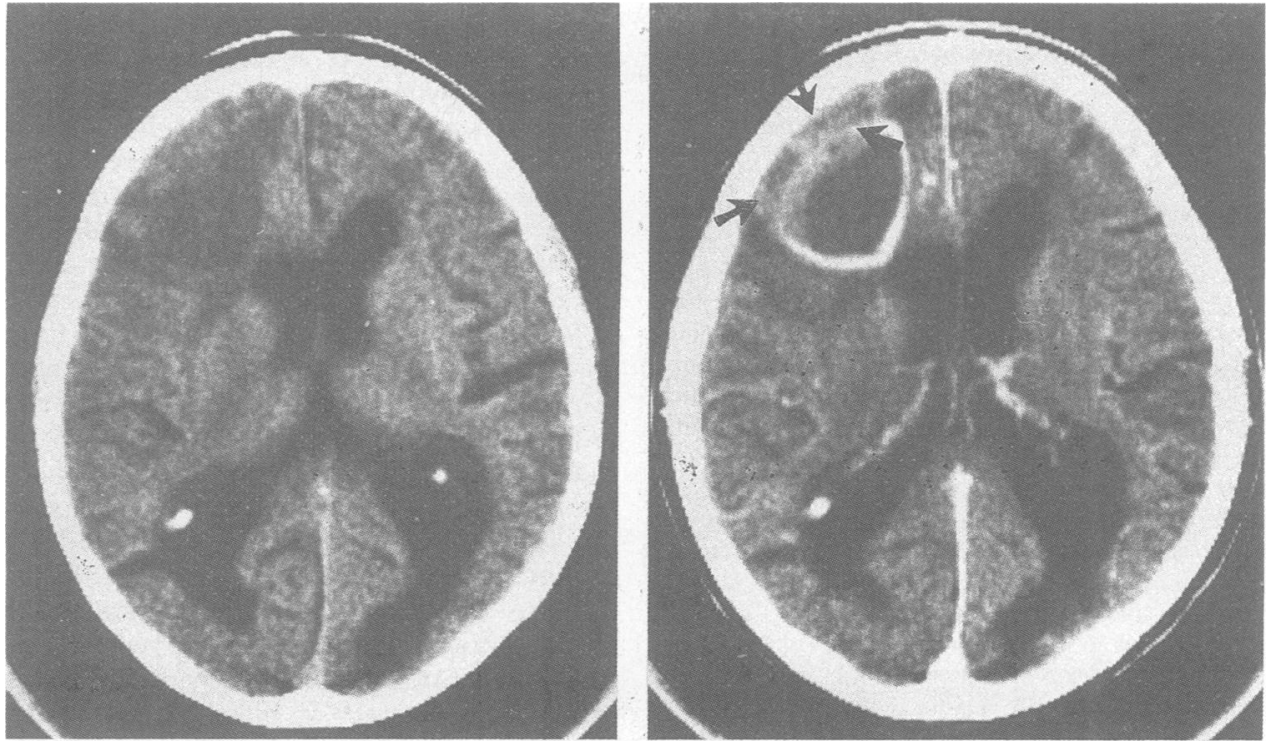

Fig 2 CT scan without contrast agent (left) demonstrates, beside an old hypodense area in the left occipital lobe, a hypodense lesion with oedema and mass effect in the right frontal lobe. After contrast enhancement (right) a ring blush and a cortical, thin, streak-like high density, close to the mass (arrows), appear.

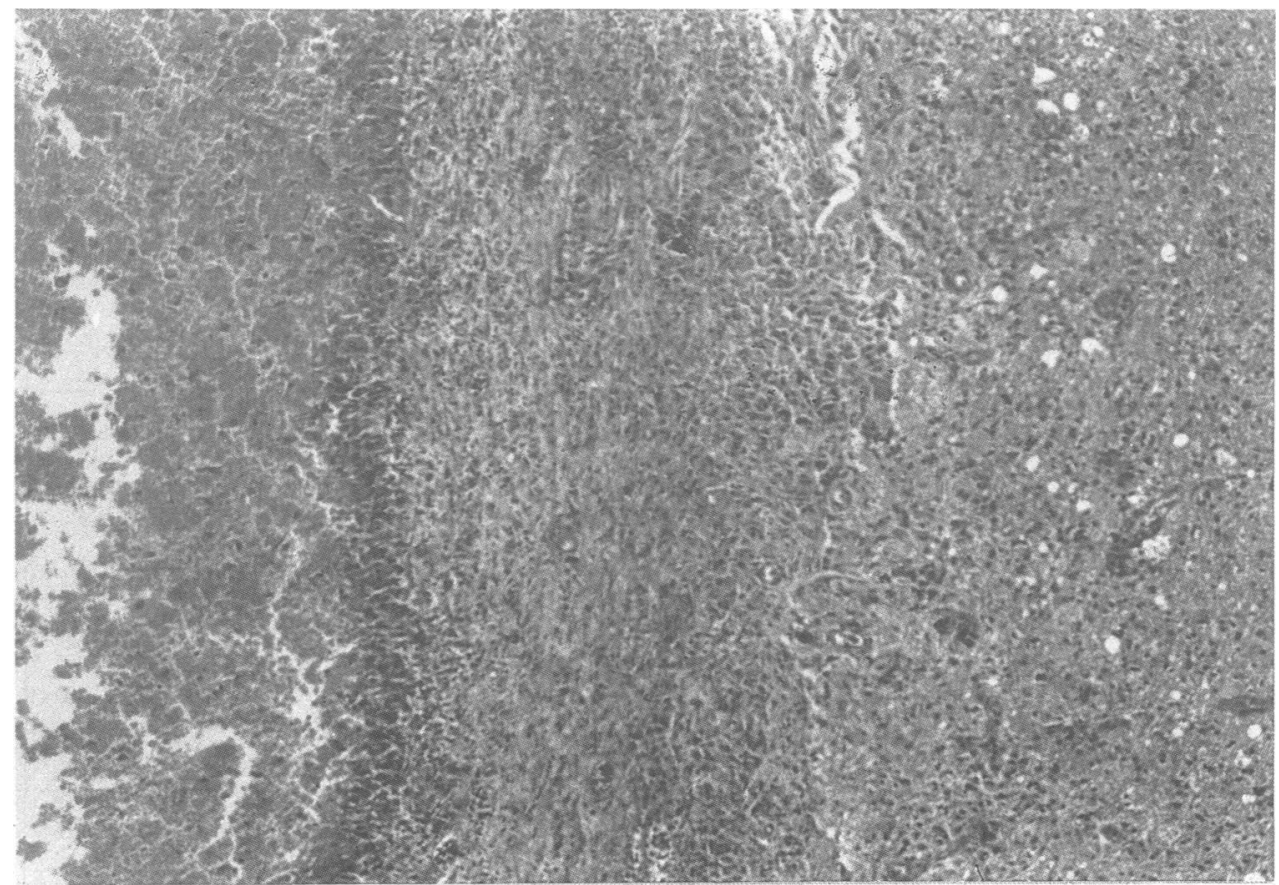

Fig 3 Photomicrograph of the capsule shows a thick, fibrous tissue, covered by cerebral tissue with reactive gliosis, on the outer side and a granulation tissue, rich in vascular channels, on the inner side. A layer of haemosiderin-laden histiocytes and necrotic red blood cells can be seen on the inner surface of the granulation tissue (Haematoxylin and eosin $\times 100$ ). 
hypodense area, suggesting ischaemia, in the left occipital lobe. There was no history of head injury or arterial hypertension.

The neurological examination on admission demonstrated a frontal syndrome and a right homonymous hemianopsia. A CT scan showed, beside the old occipital hypodense area, a low density lesion in the right frontal lobe with perifocal oedema and mass effect. An enhanced CT scan showed a ring blush and a cortical, thin, streak-like hyperdensity, close to the mass (fig 2). Right carotid angiography demonstrated an avascular space occupying lesion in the frontal lobe. The patient had a right frontal craniotomy. The first and second frontal gyri were covered by a thin, close-fitting arachnoid, yellowish membrane, that appeared like the internal membrane of a chronic subdural haematoma. About $5 \mathrm{~mm}$ below the cortex, a thick, elastic, brownish capsule was found. A small amount of dense, dark bloody fluid was aspirated by puncture. The capsule was embedded in the white matter and close to a normal cerebral artery in the depth of a sulcus. After separation from the surrounding structures, the mass was completely removed. There was no evidence of a vascular malformation or neoplasia. The capsule was sectioned: its contents consisted of clots in various stages of formation. The postoperative course was uneventful. Six months later a slight frontal syndrome remained.

\section{Histological examination}

A histological study of both surgical specimens revealed that the capsule consisted of granulation tissue rich in vascular channels on the inner side and collagenous and fibrous tissue on the outer side. Close to the inner surface of the granulation tissue a thin layer of haemosiderin-laden macrophages and necrotic red blood cells was found. Small fragments of cerebral tissue at the periphery of the lesion showed reactive gliosis (fig 3). No inflammatory or neoplastic changes were noted. There was no evidence of vascular malformation.

\section{Discussion}

In 1978 Yashon and Kosnik ${ }^{1}$ reported 12 patients who had a chronic ICH and were treated surgically. However, a true capsule surrounding the clots was not found in any patient. In $1981 \mathrm{Hirsh}$ et $a^{2}$ described two cases of chronic encapsulated ICH. Since then 16 more cases have been reported..$^{3-8}$ Our two cases presented clinical, neuroradiological, and histological features of chronic encapsulated ICH that agree with other reports. However, the surgical finding of a thin, close fitting arachnoid membrane in the second case was unusual. This, in our opinion, followed the partial "organisation" of a thin subdural haemorrhage that occurred together with the superficial encapsulated ICH.

Based on a review of the literature, we believe that two types of chronic ICH can be distinguished: (1) "non-encapsulated" and (2) encapsulated. A nonencapsulated ICH presents clinically with the sudden onset of neurological deficits which subsequently remain unchanged or improve and only seldom worsen. CT scan reveals a variable density lesion with possible slight rim enhancement and with possible mild mass effect. At operation, a chronic, liquid haematoma, without capsule, can be found.

An encapsulated ICH clinically simulates a tumour (gradual onset, slowly progressive neurological deficits or seizures). CT shows a variable density lesion, which may sometimes be calcified. It often shows ring blush and always causes a mass effect. A thick, fibrous capsule, including clots at various stages of formation, is the specific surgical and histological finding. In our opinion, it is very important to distinguish between these two types of chronic $\mathrm{ICH}$, in order to select the appropriate treatment. A nonencapsulated ICH is a truly liquid haematoma: the clot resorbs. Therefore, we believe that the treatment of this lesion can be conservative because the natural evolution generally leads to spontaneous resolution of the mass. The encapsulated ICH, on the other hand, is an expanding lesion as a result of repeated bleedings from newly formed small vessels of the inner layer of the capsule. Surgery is therefore the treatment of choice.

In some cases it can be very difficult to differentiate between these two types of chronic ICH. In such cases the best choice of treatment is to wait, since in patients with a non-encapsulated ICH serial CT scans wi $\$$ show a decrease in the size and radiographic attenus ation of the lesion, disappearance of any mass effecti and concentric reduction or disappearance of the enhancement of the rim..$^{10}$

With the formation of the capsule, Hirsh et ald believe that the capsule is formed from the fibroblast related to the abnormal vessels, which are demor strated in pathological specimens. However, in most encapsulated ICH vascular malformations are not found.

On the basis of our surgical findings, (Case 2) of a small subdural haemorrhage and an encapsulated ICH adjacent to leptomeninges, we think that, whatever the cause of the initial bleeding, the capsule formation is due to a vigorous, proliferative reaction of arachnoidal fibroblasts. This hypothesis seems to be confirmed by the fact that almost all reported encapsulated ICH are located in the superficial white matter, close to the arachnoid. The relationship with leptomeninges may not always be so clear when we consider the less superficial encapsulated ICH. However, in these cases the lesion also appears to be very near to the deep sulcal arachnoid, as in Case 1. Furthermore, it is well known that in some brain neoplasms (gliomas, desmoplastic medulloblastoma, metastasis), invasion of the leptomeninges results in marked alterations of the tumoural architecture which becomes firmer, owing to intense proliferation of connective tissue from fibroblasts of the stimulated arachnoid. ${ }^{11}$ In our opinion, both this behaviour of 
some tumours and the capsule formation of chronic ICH result from the same pathogenetic factor: an exuberant proliferation of arachnoidal fibroblasts. Nevertheless, why this exuberant proliferation occurs in only rare cases of superficial ICH remains unknown.

\section{References}

1 Yashon D, Kosnik E. Chronic intracerebral hematoma. Neurosurgery 1978;2:103-6.

2 Hirsh LF, Spector HB, Bogdanoff BM. Chronic encapsulated intracerebral hematoma. Neurosurgery 1981; 9:169-72.

3 Takahashi N, Kikuchi H, Kobayashi K, Karasawa J. Multilocular encapsulated intracerebral hematoma. Neurol Surg 1983;11:739-43.

4 Aoki N, Mizuguchi $\mathrm{K}$. Chronic encapsulated intracerebellar hematoma in infancy: case report. Neurosurgery 1984;14:594-7.
5 Lin SZ, Shih CJ, Wang YC, Tsai SH. Intracerebral hematoma simulating a new growth. Surg Neurol 1984; 21:459-64.

6 Terada T, Okuno T, Moriwaki H, Nakai E, Komai N. Chronic encapsulated intracerebral hematoma during infancy: case report. Neurosurgery 1985;16:853-5.

7 Masuzawa T, Saito K, Shimabukuro H, Iwasa H, Sato F. Chronic encapsulated hematomas in the brain. Acta Neuropathol (Berl) 1985;66:24-8.

8 Pozzati E, Giuliani G, Gaist G, Piazza G, Vergoni G. Chronic expanding intracerebral hematoma. $J$ Neurosurg 1986;65:611-4.

9 Zimmerman RD, Leeds NE, Naidich TP. Ring blush associated with intracerebral hematoma. Radiology 1977;122:707-11.

10 Tagasugi S, Ueda S, Matsumoto K. Chronological changes in spontaneous intracerebral hematoma. An experimental and clinical study. Stroke 1985;16:651-8.

11 Rubinstein LJ. Tumours of the central nervous system. Washington: Armed Forces Institute of Pathology, 1972:35-50, 130-52, 319-32. 